trabeculations and raise the potential diagnosis of left ventricular non-compaction (LVNC). It is unclear whether the myocardial morphology is representative of LVNC or whether it represents an ethnicity related epiphenomenon to increased cardiac preload. Pregnancy is associated with a marked increase in physiological cardiac preload. This study sought to investigate the impact of increased cardiac preload on left ventricular morphology in previously healthy black and Caucasian pregnant women.

Method Between 2008 and 2010, 106 consecutive normotensive and previously healthy pregnant females (90\% Caucasian) underwent cardiac echocardiography in the third trimester. Echocardiograms were analysed for trabeculations defined as localised protrusions of the ventricular wall $\geq 3 \mathrm{~mm}$ in thickness associated with intertrabecuar recesses filled with blood from the left ventricular cavity as assessed by colour Doppler. The results were compared with 80 healthy non-pregnant females (51\% Caucasian) of similar age.

Results There was no difference in mean age (29 \pm 6 years vs 32 \pm 6 years; p: 0.278$)$ or blood pressure $(111 \pm 16 \mathrm{~mm} \mathrm{Hg}$ vs 114 $\pm 15 \mathrm{~mm} \mathrm{Hg}$; $p$ 0.664) between pregnant black females and pregnant Caucasian females.

Pregnant black females demonstrated a higher prevalence of increased left ventricular trabeculation compared with pregnant Caucasian females $(n=5 ; 45.5 \%$ vs $n=12 ; 12.6 \%$; $p$ 0.014). Pregnant black females were three times more likely to have increased left ventricular trabeculation compared with pregnant Caucasian females. In contrast none of the non-pregnant females of either ethnicity exhibited any evidence of increased left ventricular trabeculaton.

None of the black or Caucasian pregnant females with increased left ventricular trabeculation showed objective features of left ventricular systolic or diastolic dysfunction. The mean EF by Simpsons method was $56.8 \pm 12 \%$ vs $58 \pm 9.8 \%$; $=0.792$. The $E / A$ ratio was $1.47 \pm 0.33$ vs $1.30 \pm 0.44$; $\mathrm{p}$ : 0.218 and isovolumic relaxation time was $81.2 \pm 1.89 \mathrm{~ms}$ vs $80.6 \pm 15.46 \mathrm{~ms}$; p: 0.6 .

Conclusions Black pregnant females exhibited a significantly higher frequency of increased left ventricular trabeculation compared with pregnant Caucasian females with similar blood pressure in the absence objective markers of abnormal systolic or diastolic function. Based on the results of this study, it is highly likely that increased cardiac preload in heart failure is associated with an ethnically mediated myocardial response comprising of an increased number of myocardial trabeculations in black individuals and should be regarded as an epiphenomenon rather than LVNCC outside the context of familial heart failure. The hypothesis requires prosposective longitudinal evaluation.

\section{INCREASED LEFT VENTRICULAR TRABECULATION IN AFRO-CARIBBEAN INDIVIDUALS: AN INHERITED CARDIOMYOPATHY OR A PHYSIOLOGICAL RESPONSE TO INCREASED CARDIAC PRELOAD}

S Gati, K Melchiorre, M Papadakis, N Sheikh, S Ghani, A Zaidi, B Thllaganathan, S Sharma St. George's University

doi:10.1136/heartjnl-2013-304019.155

Introduction Studies in heart failure patients of Afro-Caribbean (black) origin reveal a high prevalence (up to $30 \%$ ) of myocardial 